\title{
Anharmonic double- $\gamma$ vibrations in nuclei and their description in the interacting boson model
}

\author{
J. E. García-Ramos, ${ }^{1}$ C. E. Alonso, ${ }^{1}$ J. M. Arias, ${ }^{1}$ and P. Van Isacker ${ }^{2}$ \\ ${ }^{1}$ Departamento de Física Atómica, Molecular y Nuclear, Universidad de Sevilla, Apartado 1065, 41080 Sevilla, Spain \\ ${ }^{2}$ Grand Accélérateur National d'Ions Lourds, B.P. 5027, F-14076 Caen Cedex 5, France
}

(Received 17 November 1999; published 10 March 2000)

\begin{abstract}
Double- $\gamma$ vibrations in deformed nuclei are studied in the context of the interacting boson model with special reference to their anharmonic character. It is shown that large anharmonicities can be obtained with interactions that are (at least) of three-body nature between the bosons. As an example the $\gamma$ vibrations of the nucleus ${ }_{68}^{166} \mathrm{Er}_{98}$ are studied in detail.
\end{abstract}

PACS number(s): 21.60.Fw, 21.10.Re, 21.60.Ev, 27.70.+q

Nuclear quadrupole shape oscillations can be of two types: $\beta$ or $\gamma$ vibrations [1]. The $\beta$ vibration preserves axial symmetry and a one-quantum excitation gives rise to a $K$ $=0$ band where $K$ is the projection of the angular momentum on the axis of symmetry of the nucleus. A $\gamma$ vibration breaks axial symmetry and leads to a $K=2$ band. Although their existence has been conjectured a long time ago [2], the observation and interpretation of $\beta$-vibrational $K^{\pi}=0^{+}$bands is still fraught with questions and difficulties. In contrast, $\gamma$-vibrational $K^{\pi}=2^{+}$bands are systematically observed in deformed nuclei and their properties are correspondingly better understood.

Since single- $\gamma$ vibrations are so well established, it is natural to search for double- $\gamma$ vibrations and to examine their harmonic nature (i.e., whether they occur at twice the energy of the single vibration). Two intrinsic $K=2$ quanta can be combined parallel or antiparallel and hence lead to two bands: one with $K=0$ and another with $K=4$. The experimental identification of double- $\gamma$ vibrations in deformed nuclei is difficult since they are expected to lie above the pairing gap and to mix with two-quasiparticle excitations, resulting in fragmentation and a corresponding reduction in the collectivity of the states. During the past few years, however, a steady improvement of experimental techniques has allowed the measurement of low-spin states in the energy region of interest [3-5]. This possibility has reopened the old debate on the existence of two-phonon $(\beta$ or $\gamma$ ) vibrational states and their properties. Experiments have been reported recently pointing out the existence of double- $\gamma$ vibrations in several deformed nuclei with a wide range of anharmonicities [6-11]. In particular, in Refs. [7,8] the first observation of the $K^{\pi}=0^{+}$and $K^{\pi}=4^{+}$double- $\gamma$ states in one nucleus, ${ }^{166} \mathrm{Er}$, is reported. They are observed at $1.949 \mathrm{MeV}$ and $2.029 \mathrm{MeV}$, respectively. This information is of great interest since it provides a stringent test of nuclear models; for instance, the quasiphonon nuclear model (QPNM) predicts no $K^{\pi}=0^{+}$two-phonon state below $2.5 \mathrm{MeV}$ in ${ }^{166} \mathrm{Er}$ [12]. Several calculations of two-phonon states, using either phenomenological or microscopic models, are available, particularly for ${ }^{166} \mathrm{Er}$ and ${ }^{168} \mathrm{Er}[12-17]$. One of the models employed is the interacting boson model (IBM) [18]. In the simplest version of this model, referred to as IBM-1, an even-even nucleus with $n$ valence nucleons is treated as a system of $N=n / 2$ bosons with $l=0$ ( $s$ bosons) or $l=2 \quad(d$ bosons). In the usual formulation of the model only up to two-body interactions between the bosons are taken.

What are the predictions of IBM with regard to twophonon states in deformed nuclei and their (an)harmonic nature? It was pointed out some time ago by Bohr and Mottelson [13] that the IBM-1 is unable to accommodate large anharmonicities, as observed for instance in ${ }^{168}$ Er. Subsequently, it was shown that these can be described but require $g$ bosons with $l=4$ in addition to the $s$ and $d$ bosons ( $s d g$-IBM) [16]. More recently, we reported a study of twophonon states in IBM-1 treated in the intrinsic frame [19] and showed that the IBM-1 is a harmonic model in the limit of large boson number. Anharmonicities can only exist for finite boson number and they are always small if only up to two-body interactions are considered. It was also suggested that anharmonicity in the model is linked to triaxiality. Since it is known that IBM-1 with only up to two-body interactions cannot give rise to a stable triaxial minimum, the model's capability for describing anharmonicities depends on the inclusion in the Hamiltonian of higher-order interactions, some of which are known to induce triaxial shapes [20,21].

In this article the relation between three-body interactions in IBM-1 and the anharmonicity of $\gamma$ vibrations in deformed nuclei is investigated. Although the analysis presented is not exhaustive, it is shown that anharmonic behavior can be obtained with reasonable three-body interactions. As an example, the energy and E2 transition properties of the $\gamma$ vibrations of the nucleus ${ }^{166} \mathrm{Er}$ are studied in detail. In addition, the nature of the $0_{2}^{+}$state in the same nucleus, which has been the subject of an intense debate in the past few years [22-25], is briefly discussed.

The Hamiltonian adopted in the following includes a quadrupole-quadrupole term, a rotational $\hat{L}^{2}$ term, and threebody interactions between the $d$ bosons,

$$
\begin{aligned}
\hat{H}= & -\kappa \hat{Q} \cdot \hat{Q}+\kappa^{\prime} \hat{L} \cdot \hat{L}+\sum_{k l} \theta_{l}\left(\left(d^{\dagger} \times d^{\dagger}\right)^{(k)} \times d^{\dagger}\right)^{(l)} \\
& \times\left((\widetilde{d} \times \widetilde{d})^{(k)} \times \widetilde{d}\right)^{(l)}
\end{aligned}
$$

where - denotes scalar product, $\widetilde{d}_{\mu}=(-1)^{\mu} d_{-\mu}, \hat{Q}$ is the boson quadrupole operator, and $\hat{L}$ is the angular momentum operator: 


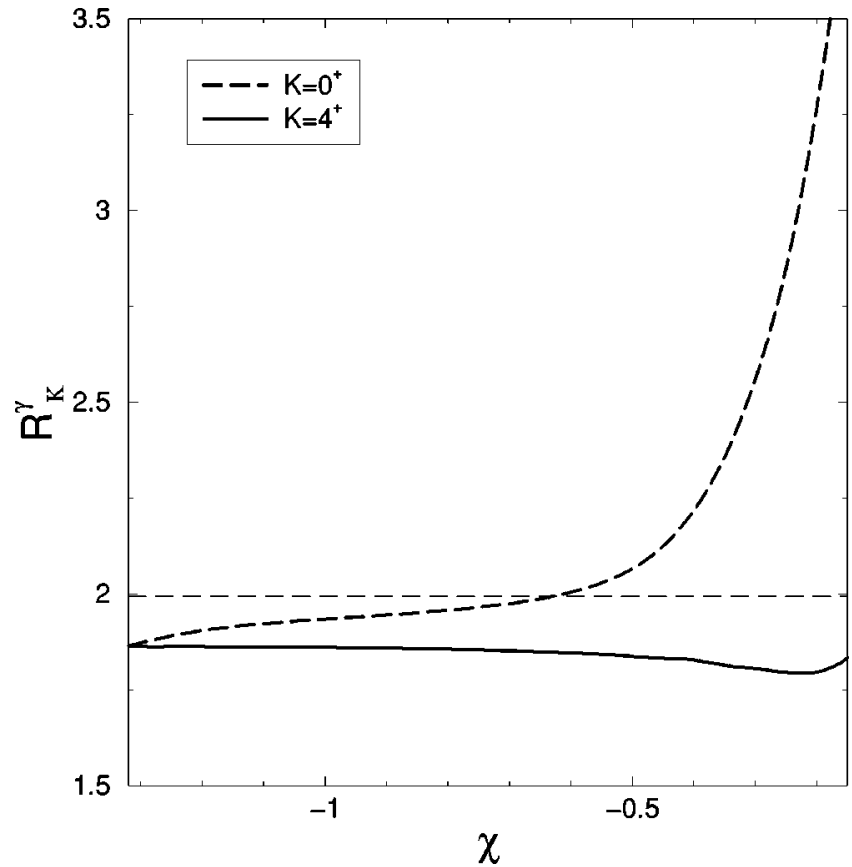

FIG. 1. The ratios $R_{K}^{\gamma}$ (as defined in the text) as a function of $\chi$. The Hamiltonian (1) is used with $\theta_{l}=0$; the boson number is $N$ $=15$.

$$
\hat{Q}=s^{\dagger} \widetilde{d}+d^{\dagger} \tilde{s}+\chi\left(d^{\dagger} \times \widetilde{d}\right)^{(2)}, \quad \hat{L}=\sqrt{10}\left(d^{\dagger} \times \widetilde{d}\right)^{(1)} .
$$

Five independent three-body $d$-boson interactions exist which have $l=0,2,3,4$, and 6 . Interactions with the same $l$ but different $k$ are not independent but differ by a normalization factor only [20]. The combinations $(k, l)=(2,0),(0,2)$, $(2,3),(2,4)$, and $(4,6)$ are chosen here.

The Hamiltonian (1) is certainly not the most general that can be considered. Notably, a vibrational term $\epsilon_{d} \hat{n}_{d}$ which dominates in spherical nuclei is omitted since it is thought of lesser importance in the deformed nuclei considered here. It is clear that the inclusion of such additional terms might improve the quality of detailed fits to particular nuclei such as the one for ${ }^{166} \mathrm{Er}$ presented below. Finally, of all possible three-body interactions only those between the $d$ bosons are retained here since these are most crucial for obtaining a stable triaxial minimum [20].

For the discussion of anharmonicities of $\gamma$ vibrations it is convenient to define the following ratios of excitation energies:

$$
R_{0}^{\gamma} \equiv \frac{E_{\mathrm{x}}\left(0_{\gamma \gamma}^{+}\right)}{E_{\mathrm{x}}\left(2_{\gamma}^{+}\right)-E_{\mathrm{x}}\left(2_{1}^{+}\right)}, \quad R_{4}^{\gamma} \equiv \frac{E_{\mathrm{x}}\left(4_{\gamma \gamma}^{+}\right)-E_{\mathrm{x}}\left(4_{1}^{+}\right)}{E_{\mathrm{x}}\left(2_{\gamma}^{+}\right)-E_{\mathrm{x}}\left(2_{1}^{+}\right)}
$$

where $0_{\gamma \gamma}^{+}$and $4_{\gamma \gamma}^{+}$are the band heads of the $K^{\pi}=0^{+}$and $K^{\pi}=4^{+}$double- $\gamma$ bands, respectively. It should be noted that the quantities $R_{K}^{\gamma}$ do not depend upon the $\hat{L}^{2}$ term in the Hamiltonian; if a single three-body term is included they depend on two parameters, $\chi$ and the ratio $\theta_{l} / \kappa$. In the present work the identification of the states $0_{\gamma \gamma}^{+}$and $4_{\gamma \gamma}^{+}$is based on the $B(E 2)$ values for decaying to the single gamma state. In Fig. 1 the quantities $R_{K}^{\gamma}$ are plotted (for $N=15$

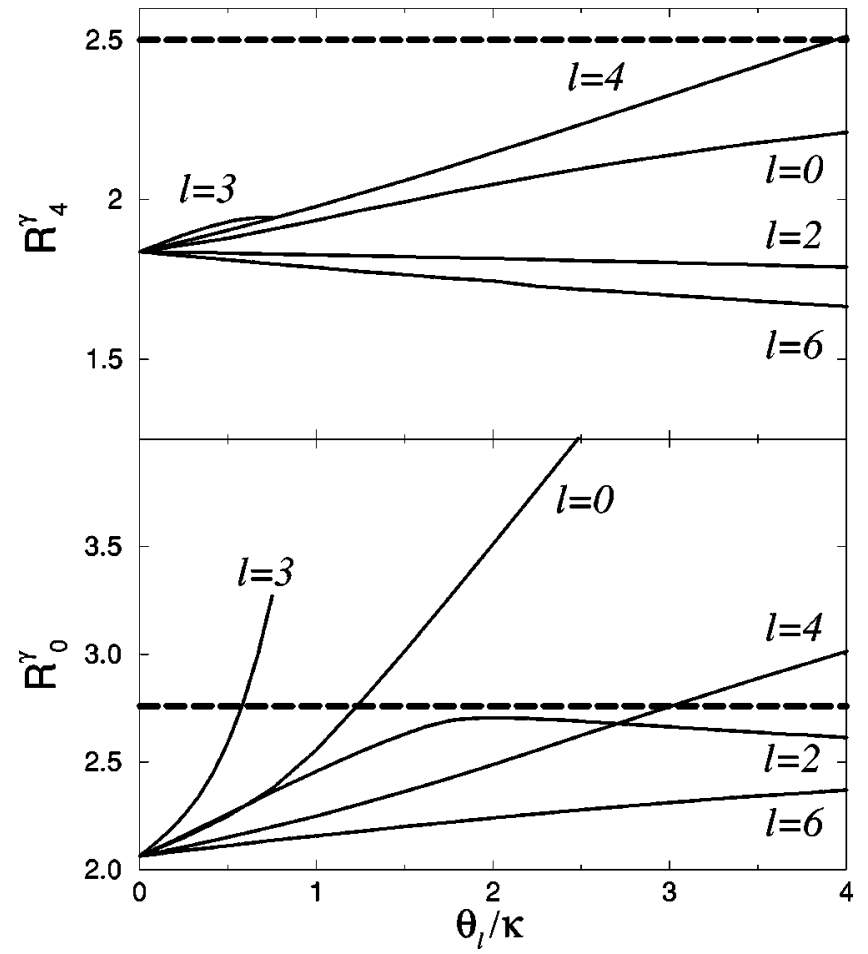

FIG. 2. The ratios $R_{K}^{\gamma}$ (as defined in the text) as a function of $\theta_{l} / \kappa$ for different $l$. The Hamiltonian (1) is used with $\chi=-0.5$; the boson number is $N=15$. The dashed lines give the experimental values for the corresponding ratios in ${ }^{166} \mathrm{Er}$.

bosons) as a function of the quadrupole parameter $\chi$ [varying between its $\mathrm{SU}(3)$ and $\mathrm{O}(6)$ values $-\frac{1}{2} \sqrt{7}$ and 0$]$ in the absence of three-body interactions. The ratio $R_{4}^{\gamma}$ remains about constant and of the order $1.8 ; R_{0}^{\gamma}$ shoots up for small $|\chi|$. Close to the $\mathrm{O}(6)$ limit the concept of a $\gamma$ vibration is not well defined and so nothing is plotted for $|\chi|<0.15$. The value of $\chi$ is constrained by $E 2$ transition probabilities and in deformed rare-earth nuclei it ranges typically between -0.4 and -0.7 [26]. From Fig. 1 it is clear that no substantial anharmonicity occurs in the $\gamma$ vibration for these values of $\chi$.

In Fig. 2 the influence of the various three-body interactions is shown for a typical value of $\chi(\chi=-0.5)$ and for $N=15$ bosons. It is seen that $\gamma$-vibrational anharmonic behavior is obtained which can be different for the $K^{\pi}=0^{+}$ and $K^{\pi}=4^{+}$bands (e.g., positive for the former while negative for the latter). Care has been taken to plot results only up to values of $\theta_{l}$ that do not drastically alter the character of rotational spectrum; beyond these values, the three-body interaction, being of highest order in the Hamiltonian (1), becomes dominant. Also shown in Fig. 2 are the ratios $R_{K}^{\gamma}$ as observed in ${ }^{166} \operatorname{Er}[7,8], R_{0}^{\gamma}=2.76$ and $R_{4}^{\gamma}=2.50$. This simple analysis shows that, on purely phenomenological grounds, the appropriate three-body interaction with the correct anharmonic character for the $K^{\pi}=0^{+}$and $K^{\pi}=4^{+}$bands in ${ }^{166} \mathrm{Er}$, has $l=4$.

Figure 3 shows the experimental spectrum of ${ }^{166} \operatorname{Er}[7,8]$ and compares it to the eigenspectrum of Hamiltonian (1) with an $l=4$ three-body interaction. The parameters are $\kappa$ 


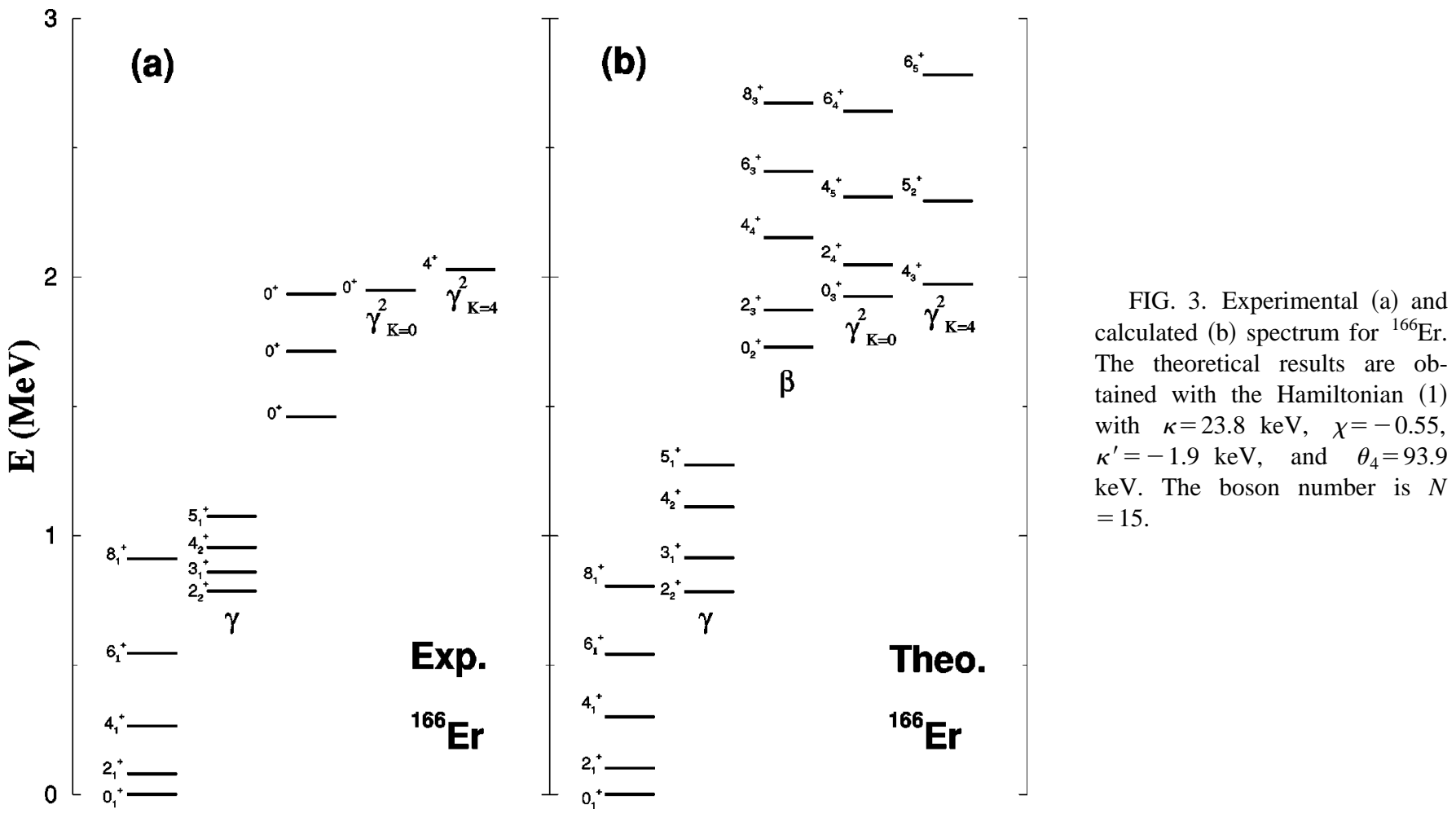

$=23.8 \mathrm{keV}, \quad \chi=-0.55, \quad \kappa^{\prime}=-1.9 \mathrm{keV}, \quad$ and $\theta_{4}$ $=93.9 \mathrm{keV}$, with boson number $N=15$. With these values the calculated excitation energies of the double- $\gamma$ band heads are $1926 \mathrm{keV}$ and $1972 \mathrm{keV}$ for the $K^{\pi}=0^{+}$and $K^{\pi}=4^{+}$ levels, respectively, leading to the ratios $R_{0}^{\gamma}=2.82$ and $R_{4}^{\gamma}$ $=2.45$, in excellent agreement with observation. Note, however, that although all $\gamma$-band heads are well reproduced by the calculation, problems arise for the moments of inertia, in particular of the $\gamma$ band. An extensive survey of combinations of cubic $d$-boson interactions has shown that it is difficult to substantially improve upon this fit although it is of course near-impossible to do an exhaustive search of the complex parameter space of all three-body interactions. In contrast, exploratory searches with simple quartic Hamilto- nians quickly yield the correct result with respect to both band-head energies and moments of inertia.

For the calculation of $E 2$ transition probabilities the consistent-Q formalism (CQF) [27] is adopted by using the E2 transition operator

$$
\hat{T}(E 2)=e_{\mathrm{eff}} \hat{Q}
$$

where $\hat{Q}$ is the boson quadrupole operator used in the Hamiltonian (1) and $e_{\text {eff }}$ is a boson effective charge, determined from the observed $B\left(E 2 ; 2_{1}^{+} \rightarrow 0_{1}^{+}\right)$value. It should be noted that the inclusion of three-body terms in the Hamiltonian would allow the use of a two-body E2 operator. However, we have not tried to do that in order to keep the calculation

TABLE I. Observed and calculated $B(E 2)$ values and ratios for ${ }^{166} \mathrm{Er}$. The $E 2$ operator (4) is used with $e_{\mathrm{eff}}^{2}=(1.83)^{2}$ W.u. and $\chi=-0.55$.

\begin{tabular}{lcc}
\hline \hline & \multicolumn{2}{c}{$B(E 2)$ value or ratio } \\
& Observed & Calculated \\
\hline$B\left(E 2 ; 2_{1}^{+} \rightarrow 0_{1}^{+}\right) \quad$ (W.u.) & $214 \pm 10^{\mathrm{a}}$ & 214 \\
$B\left(E 2 ; 4_{1}^{+} \rightarrow 2_{1}^{+}\right) \quad$ (W.u.) & $311 \pm 10^{\mathrm{a}}$ & 304 \\
$B\left(E 2 ; 2_{\gamma}^{+} \rightarrow 0_{1}^{+}\right)$(W.u.) & $5.5 \pm 0.4^{\mathrm{a}}$ & 5.3 \\
$B\left(E 2 ; 0_{2}^{+} \rightarrow 2_{1}^{+}\right) / B\left(E 2 ; 2_{\gamma}^{+} \rightarrow 0_{1}^{+}\right)$ & $0.49 \pm 0.19^{\mathrm{b}}$ & 0.21 \\
$B\left(E 2 ; 0_{2}^{+} \rightarrow 2_{\gamma}^{+}\right) / B\left(E 2 ; 2_{\gamma}^{+} \rightarrow 0_{1}^{+}\right)$ & $0.44 \pm 0.13^{\mathrm{b}}$ & 6.2 \\
$B\left(E 2 ; 0_{\gamma \gamma}^{+} \rightarrow 2_{\gamma}^{+}\right) / B\left(E 2 ; 2_{\gamma}^{+} \rightarrow 0_{1}^{+}\right)$ & $3.8 \pm 1.3^{\mathrm{c}}\left(2.2^{+1.1}-0.7^{\mathrm{d}}\right)$ & 3.2 \\
$B\left(E 2 ; 4_{\gamma \gamma}^{+} \rightarrow 2_{\gamma}^{+}\right) / B\left(E 2 ; 2_{\gamma}^{+} \rightarrow 0_{1}^{+}\right)$ & $1.3 \pm 0.4^{\mathrm{c}}\left(0.9 \pm 0.3^{\mathrm{d}}\right)$ & 2.5 \\
\hline
\end{tabular}

${ }^{\mathrm{a}}$ From Ref. [28].

${ }^{\mathrm{b}}$ From Ref. [22].

${ }^{\mathrm{c}}$ From Ref. [8]

${ }^{\mathrm{d}}$ From Ref. [7]. 
on the anharmonicity of the double-gamma excitation as simple as possible. In Table I the observed $B(E 2)$ values and ratios concerning the $\gamma$ vibrational band heads in ${ }^{166} \mathrm{Er}$ are summarized and compared to the theoretical results obtained with $e_{\text {eff }}^{2}=(1.83)^{2}$ Weisskopf units (W.u.). A good overall agreement is found but for the decay of the $0_{2}^{+}$state: the $B\left(E 2 ; 0_{2}^{+} \rightarrow 2_{\gamma}^{+}\right)$value is overpredicted by more than an order of magnitude while the $B\left(E 2 ; 0_{2}^{+} \rightarrow 2_{1}^{+}\right)$value is too small by a factor two. This casts doubt on the interpretation of the $0_{2}^{+}$observed at $1460 \mathrm{keV}$ as the $\beta$-band head. Previous interpretations of this state are contradictory: it is considered as the $\beta$-band head in [1] but as a two-quasiparticle state in [22] while Casten and von Brentano [23] claim it is a collective phonon excitation built on the $\gamma$ band. Other $0^{+}$ states are found in ${ }^{166} \mathrm{Er}$ at slightly higher energy [22] but none has the decay pattern in agreement with the present calculation. A possible explanation is that collective strength is fragmented through mixing with two-quasiparticle states which are absent from the IBM-1 model space.

In summary, three-body interactions in the IBM-1 can account for a wide variety of $\gamma$-vibrational anharmonicities in nuclei such as for instance those observed in ${ }^{166} \mathrm{Er}$ but not without substantially changing the moments of inertia of various bands. The knowledge of the two double- $\gamma$ vibrational bands $\left(K^{\pi}=0^{+}\right.$and $\left.K^{\pi}=4^{+}\right)$in a single nucleus provides a stringent test of nuclear models and, specifically, of the type and strength of three-body interactions in IBM-1. More experiments on double- $\gamma$ vibrations are thus called for since they should provide essential information concerning the systematic behavior of these states and hence the interactions involved. From the theoretical side, a systematic analysis of all three-body interactions and not just those between the $d$ bosons seems in order. Once a fuller knowledge is acquired of the systematic behavior of the interactions necessary to reproduce the observed anharmonicities, one may then attempt an understanding on a microscopic level.

We are grateful to F. Iachello, A. Vitturi, and C. Volpe for valuable comments. This work has been supported in part by the Spanish DGICYT under Contract No. PB98-1111 and by one IN2P3 (France)-CICYT (Spain) agreement.
[1] A. Bohr and B. R. Mottelson, Nuclear Structure, Vol. II (Benjamin, Reading, MA, 1975).

[2] A. Bohr and B. R. Mottelson, Mat. Fys. Medd. K. Dan. Vidensk. Selsk. 27, 16 (1953).

[3] C. Fahlander et al., Nucl. Phys. A485, 327 (1988).

[4] H. G. Börner and J. Jolie, J. Phys. G 19, 217 (1993).

[5] T. Belgya, G. Molnár, and S. W. Yates, Nucl. Phys. A607, 43 (1996).

[6] H. G. Börner, J. Jolie, S. J. Robinson, B. Krusche, R. Piepenbring, R. F. Casten, A. Aprahamian, and J. P. Draayer, Phys. Rev. Lett. 66, 691 (1991).

[7] C. Fahlander, A. Axelsson, M. Heinebrodt, T. Härtlein, and D. Schwalm, Phys. Lett. B 388, 475 (1996).

[8] P. E. Garrett, M. Kadi, M. Li, C. A. McGrath, V. Sorokin, M. Yeh, and S. W. Yates, Phys. Rev. Lett. 78, 4545 (1997).

[9] A. Guessous et al., Phys. Rev. Lett. 75, 2280 (1995).

[10] A. Aprahamian, X. Wu, S. M. Fischer, W. Reviol, and J. X. Saladin, in Proceedings of the 8th International Symposium on Capture Gamma-Ray Spectroscopy, edited by J. Kern (World Scientific, Singapore, 1994), p. 57.

[11] F. Corminboeuf, J. Jolie, H. Lehmann, K. Fohl, F. Hoyler, H. G. Börner, C. Doll, and P. E. Garrett, Phys. Rev. C 56, R1201 (1997).

[12] V. G. Soloviev, A. V. Sushkov, and N. Yu. Shirikova, Phys. Rev. C 51, 551 (1995).

[13] A. Bohr and B. R. Mottelson, Phys. Scr. 25, 28 (1982).
[14] T. S. Dumitrescu and I. Hamamoto, Nucl. Phys. A383, 205 (1982).

[15] M. K. Jammari and R. Piepenbring, Nucl. Phys. A487, 77 (1988).

[16] N. Yoshinaga, Y. Akiyama, and A. Arima, Phys. Rev. Lett. 56, 1116 (1986); Phys. Rev. C 38, 419 (1988).

[17] V. G. Soloviev, A. V. Sushkov, and N. Yu. Shirikova, J. Phys. G 20, 113 (1994).

[18] F. Iachello and A. Arima, The Interacting Boson Model (Cambridge University Press, Cambridge, England, 1987).

[19] J. E. García-Ramos, C. E. Alonso, J. M. Arias, P. Van Isacker, and A. Vitturi, Nucl. Phys. A637, 529 (1998).

[20] P. Van Isacker and J. Q. Chen, Phys. Rev. C 24, 684 (1981).

[21] K. Heyde, P. Van Isacker, M. Waroquier, and J. Moreau, Phys. Rev. C 29, 1420 (1984).

[22] P. E. Garrett, M. Kadi, C. A. McGrath, V. Sorokin, M. Li, M. Yeh, and S. W. Yates, Phys. Lett. B 400, 250 (1997).

[23] R. F. Casten and P. von Brentano, Phys. Rev. C 50, R1280 (1994); 51, 3528 (1995).

[24] D. G. Burke and P. C. Sood, Phys. Rev. C 51, 3525 (1995).

[25] C. Günther, S. Boehmsdorff, K. Freitag, J. Manns, and U. Muller, Phys. Rev. C 54, 679 (1996).

[26] R. F. Casten and D. D. Warner, Rev. Mod. Phys. 60, 389 (1988).

[27] D. D. Warner and R. F. Casten, Phys. Rev. Lett. 48, 1385 (1982).

[28] E. N. Shurshikov and N. V. Timofeeva, Nucl. Data Sheets 67, 45 (1992). 